\title{
On an Angle with Magical Properties
}

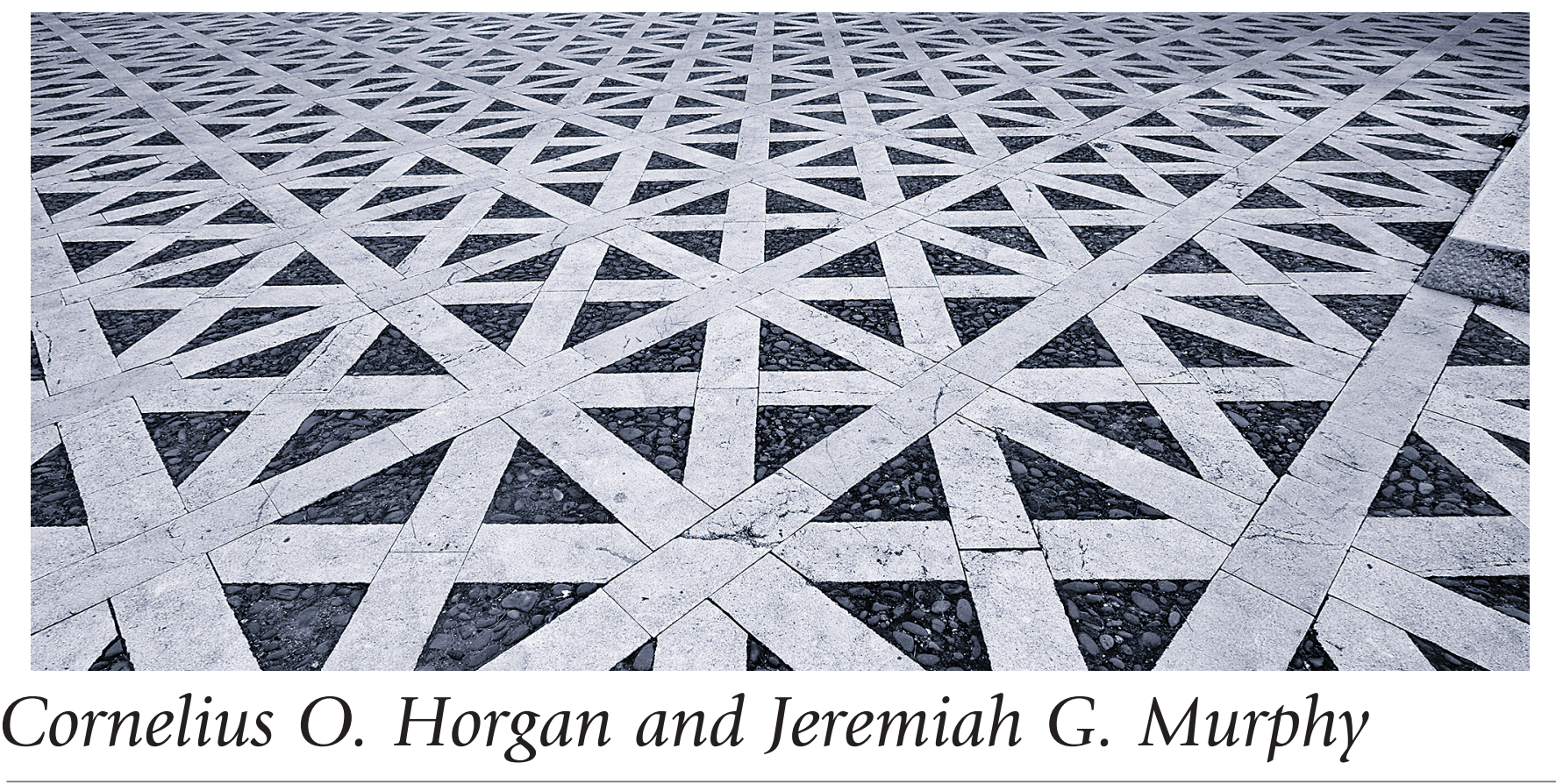

\section{Introduction}

The angle defined by

$$
\begin{aligned}
\theta_{m} & =\arctan (\sqrt{2})=\arcsin (\sqrt{2 / 3}) \\
& =\arccos (1 / \sqrt{3})=54.74^{\circ}
\end{aligned}
$$

arises in a surprising number of diverse application areas in science and engineering which seem to have very little connection to one another. Its ubiquitous nature has led to it being called a "magic angle." In this expository short article, we provide an outline of why such nomenclature is warranted and hope to draw the attention of the mathematics and science communities to this intriguing concept.

One of the earliest findings regarding a "magic angle" concept arose in connection with hydrostatic skeletons or muscular hydrostats such as the common worm, octopus

Cornelius O. Horgan is the Wills Johnson Professor of Applied Mathematics and Mechanics Emeritus, School of Engineering and Applied Science at the University of Virginia. His email address is coh8p@virginia.edu.

Jeremiah G. Murphy is a professor of mechanical engineering at Dublin City University, Ireland. His email address is jeremi ah.murphy@dcu. i e.

Communicated by Notices Associate Editor Reza Malek-Madani.

For permission to reprint this article, please contact:

reprint-permission@ams.org.

DOI: https://doi.org/10.1090/noti2398 arm, or elephant trunk. Such hydrostats are characterized by cylindrical lattice structures composed of families of inextensible helically wound fibers (modeling fibers of stiff collagen arranged in alternate left- and right-handed geodesic helices). Special angles also occur in analysis of the mechanical behavior of fiber-reinforced incompressible nonlinearly elastic soft solids. In this context, the magic angle concept occurs most commonly in structural elements composed of circular cylindrical tubes or solid cylinders reinforced by helically wound fibers, but also occurs in flat thin sheets reinforced by fibers in the plane. The fibers can be inextensible as in reinforced rubber or extensible such as collagen fibers in soft tissue. Fibers orientated at the magic angle give rise to special mechanical responses. Magic angles also arise in the field of soft robotics in connection with artificial muscles as well as in nuclear magnetic resonance. In this short exposition, we highlight some of the most interesting results on magic angles. The interested reader is directed to the references cited for details. 

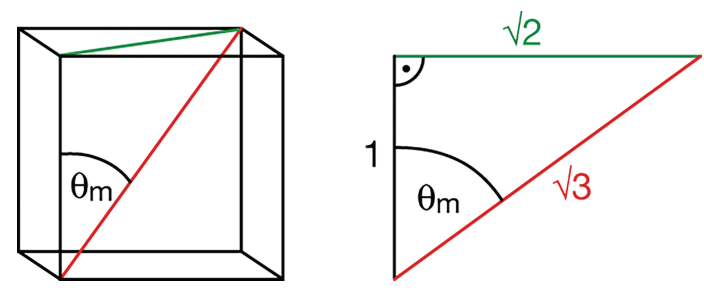

Figure 1. Geometry of magic angle.

\section{Geometry}

The magic angle is defined by (1.1) and is often characterized in various applications as the smallest angle for which

$$
2 \cos ^{2} \theta-\sin ^{2} \theta=0
$$

or equivalently, by

$$
3 \cos ^{2} \theta-1=0 \text { or } 2-3 \sin ^{2} \theta=0 .
$$

Sometimes the "magic angle" terminology is used for the complement $\theta_{m c}$ of this angle $\left(90^{\circ}-54.74^{\circ}=35.26^{\circ}\right)$ so that

$$
\begin{aligned}
\theta_{m c} & =\arctan (1 / \sqrt{2})=\arccos (\sqrt{2 / 3}) \\
& =\arcsin (1 / \sqrt{3})=35.26^{\circ}
\end{aligned}
$$

and in this case the analogs of (2.1) and (2.2) are

$$
\cos ^{2} \theta-2 \sin ^{2} \theta=0
$$

and

$$
3 \sin ^{2} \theta-1=0, \quad 2-3 \cos ^{2} \theta=0,
$$

respectively. We will call the angle (2.3) the "complementary magic angle."

The angle (1.1) can be given a direct geometric characterization. An obvious one is that depicted in the right angle triangle shown on the right in Figure 1. Moreover, from Figure 1, we see that $\theta_{m}$ is the angle between the space diagonal of a unit cube and any of its three connecting edges. It is also half of the opening angle formed when a cube is rotated from its space diagonal axis, which may be represented as $\arccos (-1 / 3)$ or $2 \arctan (\sqrt{2})=109.4712^{\circ}$. This double magic angle is directly related to tetrahedral molecular geometry and is the angle from one vertex to the exact center of a tetrahedron (the tetrahedral angle).

A nongeometric way of characterizing the angle (1.1) is as the first zero of the function

$$
F(\theta)=P_{2}(\cos \theta)=\frac{1}{2}\left(3 \cos ^{2} \theta-1\right),
$$

where $P_{2}(\cos \theta)$ is the second-order Legendre polynomial.

\section{Biology}

The paper by Goriely and Tabor [1] and the book by Goriely [2] provide an informative summary of the various contexts in biology where the magic angle concept arises. As pointed out there, apparently one of the first studies where the special angle (1.1) was encountered was in a seminal paper in 1952 by Cowey [3] concerned with the locomotion and flattening of worms. For a circular cylindrical lattice structure reinforced by a double family of inextensible helically wound fibers, it was shown there that the volume enclosed by a single turn of the helical system is

$$
V=\frac{D^{3} \sin ^{2} \theta \cos \theta}{4 \pi}
$$

where $D$ denotes the constant length of one fiber turn and $\theta$ is the pitch angle. See Goriely [2] and Horgan and Murphy [4], [9] for further details. The maximum volume occurs when $\frac{d V}{d \theta}=0$ which yields equation (2.1), i.e., the maximum volume occurs at the magic angle $\theta_{m}$. This classic result for hydrostats was obtained based solely on geometric considerations. Further developments are described in Clark and Cowey [5] who considered the case of collagen fiber extensibility when now the volume of soft tissue enclosed is constant. In this case, (3.1) can be rearranged to give

$$
D=(4 \pi V)^{1 / 3} \sin ^{-2 / 3} \theta \cos ^{-1 / 3} \theta
$$

and it is easy to verify that $D$ has a minimum at the magic angle $\theta_{m}$.

The paper by Kim and Segev [6] describes how the intriguing mechanics of an octopus arm also give rise to a magic angle. The octopus arm, like the elephant trunk, is an example of a muscular hydrostat. As pointed out in [1], [2], another example in biology where the magic angle occurs is in the study of elongation of notochords of vertebrate embryos. The magic angle also arises in the field of soft robotics in connection with McKibben actuators that can serve as artificial muscles (see [1], [2] for pertinent references).

\section{Mechanics of Elastic Fibrous Soft Materials}

There has been considerable recent interest in the role played by magic angles in the mechanics and physics of fiber-reinforced nonlinearly elastic materials. The applications involving such materials have classically been in connection with rubber and in particular in the design of reinforced rubber tubes and hoses. It has also been demonstrated in recent years that concepts from continuum mechanics have widespread application in the biomechanics of soft tissues where the fiber reinforcement is now due to collagen fibers in a matrix of elastin. The work of Demirkoparan and Pence [7] is concerned with circular cylindrical hyperelastic tubes reinforced by a symmetric doubly helically wound family of extensible fibers. See Figure 2 for a typical layout.

The tubes are subject to the combined effects of internal pressure and interior wall swelling. A more general framework for fiber reinforcement, but one that does not include wall swelling, was also considered in [1] where nonsymmetric fiber families were treated as well as the effect of 


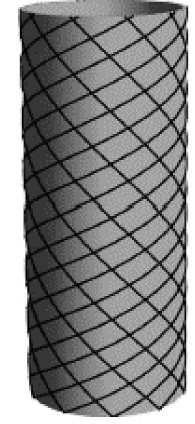

Figure 2. A doubly helically wound fiber-reinforced hyperelastic tube.

prestretch of the fibers. Both papers use a theory of nonlinear elasticity for orthotropic materials. It is shown how the magic angle separates different response modes in the fiber-reinforced body. In particular, a pressurized tube reinforced by a doubly symmetric family of helically wound fibers contracts in length and expands radially if the fibers are wound at an angle smaller than the magic angle while the tube increases in length and contracts radially if the fibers are wound at an angle greater than the magic angle. As was pointed out in [7], [4], in thin-walled closed cylindrical pressure vessels, the maximum strength is obtained when the ratio of the hoop (circumferential) stress to axial stress is 2:1 which occurs at the magic angle. The magic angle is the optimal winding angle for the design of filament-wound structures and is often derived in the composites structures literature by what is known as "netting analysis." Further results with applications to soft robots are described in [15]. It was pointed out in [7] that the synthetic fibers in fire hoses are aligned at the magic angle to minimize sudden jerk as the hose is suddenly turned on. Similarly, the spray hoses in kitchen sinks and the common garden hose are generally reinforced with helical fibers orientated at this angle (see Figure 3).

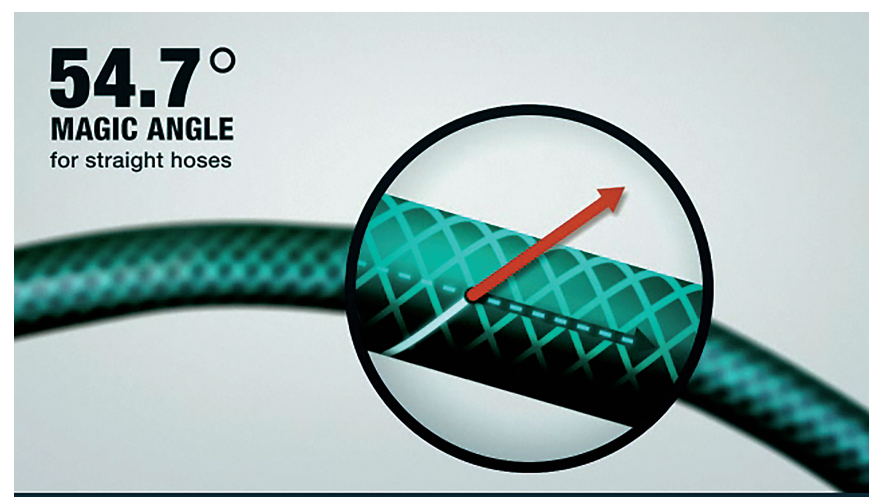

Figure 3. The common garden hose.

Several further results in the mechanics of fibrous incompressible elastic materials are described in [4], [8], [9] and references cited therein. It was shown in [4] that fibers orientated at the magic angle result in quasi-isotropic mechanical response of fiber-reinforced composites. Furthermore, a generalization of the magic angle concept is given as the angle for which the fiber stretch is zero. For both transversely isotropic and orthotropic fiber-reinforced materials, it is shown that fiber compression can occur at the magic angle resulting in material instability. A generalization of the magic angle to the nonlinear deformation regime is also proposed in [4] where the characterization given in (2.2) is generalized to

$$
\cos ^{2} \theta=\frac{1}{1+\lambda+\lambda^{2}},
$$

where $\lambda \geq 1$ denotes a stretch. The specialization to infinitesimal deformations is obtained on letting $\lambda \rightarrow 1$ in (4.1) so that one recovers the classical magic angle (1.1) in this limit.

The potential occurrence of a magic angle in the collagen fiber orientation of the coronary arterial wall has also received some previous attention in the literature (see the discussion in [9]).

\section{Other Applications}

As was remarked in [1], the term "magic angle" was introduced there to reflect the appearance "as if by magic" in several different settings in biology, mechanics, and physics. Furthermore, as was pointed out there and in [2], it turns out that this terminology was also proposed independently much earlier in a completely different context, namely in solid-state nuclear magnetic resonance. As described in [10], [11], in nuclear magnetic resonance (NMR) spectroscopy, the orientation of the interaction tensor with the external magnetic field plays a major role. By spinning the sample around a given axis, the average angular dependence has a simple expression in terms of the angle of the axis of rotation relative to the magnetic field (see [9], [11] for details). When this angle, which is at the experimentalist's disposal, is set equal to the magic angle (1.1), then the average angular dependence vanishes (see Figure 4). It is in this context that the characterization of the magic angle in terms of the first zero of the second-order Legendre polynomial (2.6) arises.

Magic angle spinning is a technique in solid-state NMR spectroscopy which employs this principle to remove or reduce the influence of anisotropic interactions, thereby increasing spectral resolution (see, e.g., Hennel and Klinowski [10], Alia et al. [11] for details). The review article [10] provides numerous illustrations of magic angle imaging. According to Hennel and Klinowski [10], the name "magic angle spinning" in NMR was originally suggested by the late Professor Gorter of Leiden at the AMPERE congress in Pisa in 1960. A recent article in Skeletal Radiology [12] is concerned with clarifying common misconceptions among practicing radiologists regarding magic angle 


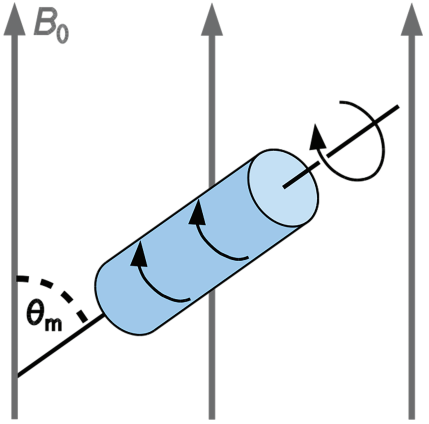

Figure 4. Magic angle spinning in NMR $\left(B_{0}\right.$ is the magnetic field vector).

imaging. Novel applications of magic angle spinning in the field of food science are described by Jensen and Bertram [13].

We conclude by pointing out yet another application of the magic angles (1.1) and (2.3) in a completely different physical context, namely in the analysis of plasticity of metals. In tensile testing of wide flat bars, it has long been established that localized necking (a sudden rapid decrease in the lateral dimension) occurs along a line orientated at the angle $\arctan (\sqrt{2})$ with respect to the tensile axis. This angle is precisely the magic angle defined in (1.1). Moreover, as described in [14], at a crack tip in thin sheets of ductile metals, ligament lines of localized necking to the free edges are inclined at the complementary magic angle (2.3) with respect to the crack line (see Figure 1.2.8 on page 15 of [14]).

ACKNOWLEDGMENTS. The authors thank Professor Reza Malek-Madani as well as the anonymous referees for their constructive remarks on an earlier version of this article.

\section{References}

[1] Alain Goriely and Michael Tabor, Rotation, inversion and perversion in anisotropic elastic cylindrical tubes and membranes, Proc. R. Soc. Lond. Ser. A 469 (2013), no. 2153, 20130011, 19, DOI 10.1098/rspa.2013.0011. MR3031490

[2] Alain Goriely, The mathematics and mechanics of biological growth, Interdisciplinary Applied Mathematics, vol. 45, Springer, New York, 2017, DOI 10.1007/978-0-387-877105. MR3585488

[3] J. B. Cowey, The structure and function of the basement membrane muscle system in Amphiporus lactifloreus (Nemertea), Quart. J. Micr. Sci. 93 (1952), 1-15.

[4] C. O. Horgan and J. G. Murphy, Magic angles for fibrous incompressible elastic materials, Proc. R. Soc. Lond. Ser. A 474 (2018), no. 2211, 20170728, 17, DOI 10.1098/rspa.2017.0728. MR3789485

[5] R. B. Clark and J. B. Cowey, Factors controlling the change of shape of certain nemertean and turbellarian worms, J. Experimental Biol. 35 (1958), 731-748

[6] Dorian Kim and Reuven Segev, Various issues raised by the mechanics of an octopus' arm, Math. Mech. Solids 22 (2017), no. 7, 1588-1605, DOI 10.1177/1081286515599437. MR3742393

[7] H. Demirkoparan and T. J. Pence, Magic angles for fiber reinforcement in rubber-elastic tubes subject to pressure and swelling, Int. J. Nonlin. Mech. 68 (2015), 87-95.

[8] C. O. Horgan and J. G. Murphy, Magic angles and fibre stretch in arterial tissue: Insights from the linear theory, J. Mech. Behav. Biomed. Materials 88 (2018), 470-477.

[9] C. O. Horgan and J. G. Murphy, Magic angles in the mechanics of fibrous soft materials, Mechanics of Soft Materials 1 (2019), \# 2.

[10] J. W. Hennel and J. Klinowski, Magic-angle spinning: a historical perspective, New Techniques in Solid-State NMR, Springer, New York, 2005, pp. 1-14.

[11] A. Alia, S. Ganapathy, and H. J. M. de Groot, Magic-angle spinning (MAS) NMR: a new tool to study the spatial and electronic structure of photosynthetic complexes, Photosynth. Res. 102 (2009), 415-425.

[12] M. L. Richardson, B. Amini, and T. L. Richards, Some new angles on the magic angle: what MSK radiologists know and don't know about this phenomenon, Skeletal Radiology 47 (2018), 1673-1681.

[13] H. M. Jensen and H. C. Bertram, The magic angle view to food: magic angle spinning (MAS) NMR spectroscopy in food science, Metabolomics 15 (2019), art. \# 44.

[14] K. B. Broberg, Cracks and fracture, Academic Press, New York, 1999.

[15] A. Chatterjee, N. R. Chahare, P. Kondiah, and N. Gundiah, Role of fiber orientations in the mechanics of bioinspired fiber-reinforced elastomers, Soft Robotics (2021) (in press) doi.org/10.1089/soro.2019.0191

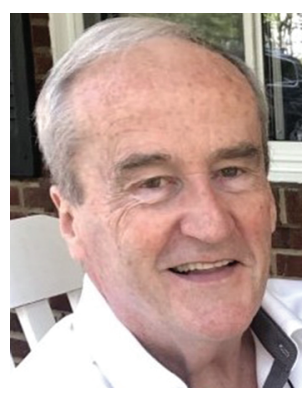

Cornelius $\mathrm{O}$. Horgan

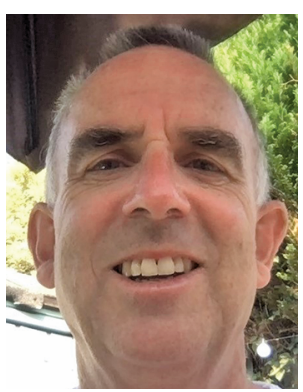

Jeremiah G. Murphy
Credits

Opening image is courtesy of Marco Rosario Venturini Autieri via Getty.

Figure 1 is courtesy of Nicoguaro via Wikimedia Commons. Licensed under CC BY-SA 4.0.

Figure 2 and photo of Cornelius O. Horgan are courtesy of Cornelius O. Horgan.

Figure 3 is courtesy of Blomaard via Wikimedia Commons. Licensed under CC BY-SA 3.0.

Figure 4 is courtesy of Dtrx via Wikimedia Commons. Licensed under CC BY-SA 3.0.

Photo of Jeremiah G. Murphy is courtesy of Jeremiah G. Murphy. 